Accepted by ApJS 2008 Jan 9; submitted 2007 Dec 3

\title{
Probing Interstellar Dust With Space-Based Coronagraphs
}

\author{
N. J. Turner, K. Grogan and J. B. Breckinridge \\ Jet Propulsion Laboratory, California Institute of Technology, Pasadena, California 91109, \\ USA; Neal.Turner@jpl.nasa.gov
}

\begin{abstract}
We show that space-based telescopes such as the proposed Terrestrial Planet Finder Coronagraph will be able to detect the light scattered by the interstellar grains along lines of sight passing near stars in our Galaxy. The relative flux of the scattered light within one arcsecond of a star at 100 pc in a uniform interstellar medium of $0.1 \mathrm{H}$ atoms $\mathrm{cm}^{-3}$ is about $10^{-7}$. The halo increases in strength with the distance to the star and is unlikely to limit the coronagraphic detection of planets around the nearest stars. Grains passing within 100 AU of Sun-like stars are deflected by radiation, gravity and magnetic forces, leading to features in the scattered light that can potentially reveal the strength of the stellar wind, the orientation of the stellar magnetic field and the relative motion between the star and the surrounding interstellar medium.
\end{abstract}

Subject headings: ISM: dust — scattering — radiative transfer — techniques: high angular resolution

\section{INTRODUCTION}

Scattered starlight is a valuable tool for measuring the properties of interstellar dust. In optical reflection nebulae (Witt et al. 1992; Calzetti et al. 1995; Burgh et al. 2002), dark clouds (Fitzgerald et al. 1976; Witt et al. 1990) and the diffuse Galactic light (Hurwitz et al. 1991; Murthy \& Henry 1995; Witt et al. 1997) the scattering grains typically lie many arcseconds from the illuminating sources. The dust can be nearer to or further from us than the source and the detected photons have been deflected through a wide range of angles. Further information about interstellar grains comes from stellar X-rays scattered by foreground

dust within a few arcseconds of the star (Mathis et al. 1995; Smith \& Dwek 1998; Witt et al. 
2001; Draine \& Tan 2003). The measurements sample the whole column of material between us and the star because the grains are strongly forward scattering at X-ray energies.

Here we show that high-contrast imaging with a space-based telescope such as the proposed Terrestrial Planet Finder Coronagraph enables a new kind of measurement for probing dust at projected separations similar to the X-ray measurements. The technique samples a range of scattering angles because at visible wavelengths the scattering is only mildly biased in the forward direction. As a consequence, most of the light comes from foreground grains lying close to the star. The scattered light halo carries information on the interstellar dust passing through the cavity opened in the interstellar gas by the stellar wind.

Evidence for the existence of interstellar grains within our own heliosphere was obtained by the Ulysses spacecraft when 55 dust impacts as measured by the Cosmic Dust Experiment were identified as interstellar by their speed, mass and arrival direction (Grün et al. 1994). The overall contribution of interstellar dust to the zodiacal cloud - the debris disk of the solar system - is unknown, although the fraction is presumably greater in the outer solar system. Collisional debris from the asteroid belt dominates inside 3 AU (Grogan et al. 2001). However the interstellar grains, charged by photoionization and strongly influenced by the solar gravity, radiation pressure and magnetic field, are able to penetrate deep into the inner solar system. A uniform incoming spatial distribution of interstellar grains becomes strongly clumped as a function of particle size and phase of the solar cycle, although the contribution of this component to the all-sky thermal emission as viewed from $1 \mathrm{AU}$ is negligible due to the local dominance of asteroidal and cometary material (Grogan et al. 1996).

Starlight scattered by interstellar dust also is a source of noise in coronagraphic planet searches. We calculate the expected brightness and distribution of the scattered light and show that the halo is unlikely to affect the direct detection of Earth-like planets around the nearest stars. We compute the trajectories of interstellar grains under the stellar gravity, radiation and magnetic forces and show that the stellar wind produces observable signatures in the scattered light. For a large number of stars at distances greater than 100 pc, highcontrast imaging can potentially yield detailed information about the stellar wind and the adjacent interstellar medium.

The remaining five sections of the paper cover the radiative transfer method (§2), the results for uniformly-distributed dust, obtained analytically assuming isotropic scattering (§3) and numerically including the anisotropy (§4), the results for dust passing through a model stellar wind ( 55$)$, and a summary and conclusions ( 66$)$. 


\section{RADIATIVE TRANSFER}

The transfer of visible light through the optically-thin interstellar medium is dominated by scattering and the radiative transfer equation at wavelength $\lambda$ is

$$
\frac{d I_{\lambda}}{d \tau_{\lambda}}=-\omega_{\lambda} \oint \Phi_{\lambda}\left(\mathbf{\Omega}^{\prime} \rightarrow \Omega\right) I_{\lambda}\left(\mathbf{\Omega}^{\prime}\right) d \mathbf{\Omega}^{\prime}
$$

where $I_{\lambda}$ is the monochromatic specific intensity, $\tau_{\lambda}$ the optical depth, $\omega_{\lambda}$ the albedo of the scattering grains and $\Phi_{\lambda}$ the phase function describing scattering from all directions $\boldsymbol{\Omega}^{\prime}$ into the beam direction $\Omega$. The star is approximated by a disk of uniform specific intensity $I_{\lambda}^{*}$, subtending a small angle $2 \psi$ as seen from a dust grain on our line of sight (figure 1). The scattering phase function is taken to be constant across the face of the star, and the solid angle filled by the star is $\pi \psi^{2}$. The transfer equation reduces to

$$
\frac{d I_{\lambda}}{d \tau_{\lambda}}=-\omega_{\lambda} \Phi_{\lambda}(\phi) \pi \psi^{2} I_{\lambda}^{*}
$$

where $\phi$ is the scattering angle between the rays joining star to grain and grain to observer. The intensity of the scattered light is calculated by integrating eq. 2 along the line of sight. The angles $\phi$ and $\psi$ vary with the distance from the observer, $z$. Writing the distance in place of the optical depth using $d \tau_{\lambda}=\chi_{\lambda} \rho d z$, neglecting emission from any distant background, and taking the simple case of an interstellar medium with uniform opacity, the intensity is

$$
I_{\lambda}=\chi_{\lambda} \rho \omega_{\lambda} I_{\lambda}^{*} \int_{0}^{\infty} \Phi_{\lambda}(\phi) \pi \psi^{2} d z
$$

\section{ISOTROPIC SCATTERING}

We first solve equation 3 assuming isotropic scattering, with $\Phi_{\lambda}=1 /(4 \pi)$ independent of $\phi$ and $z$. Along a line of sight offset by a small angle $\theta$ from the direction to the star, the stellar angular radius $\psi$ varies as

$$
\psi(z)=\frac{r_{*} / D}{\left(\theta^{2}+[1-z / D]^{2}\right)^{1 / 2}},
$$

where the star has radius $r_{*}$ and lies at distance $D$. With a change of variable to $y=$ $(1-z / D) / \theta$, the solution to the transfer equation is

$$
\frac{I_{\lambda}(\theta)}{I_{\lambda}^{*}}=\frac{\chi_{\lambda} \rho \omega_{\lambda} r_{*}^{2}}{4 D \theta} \int_{-\infty}^{1 / \theta} \frac{d y}{1+y^{2}} .
$$




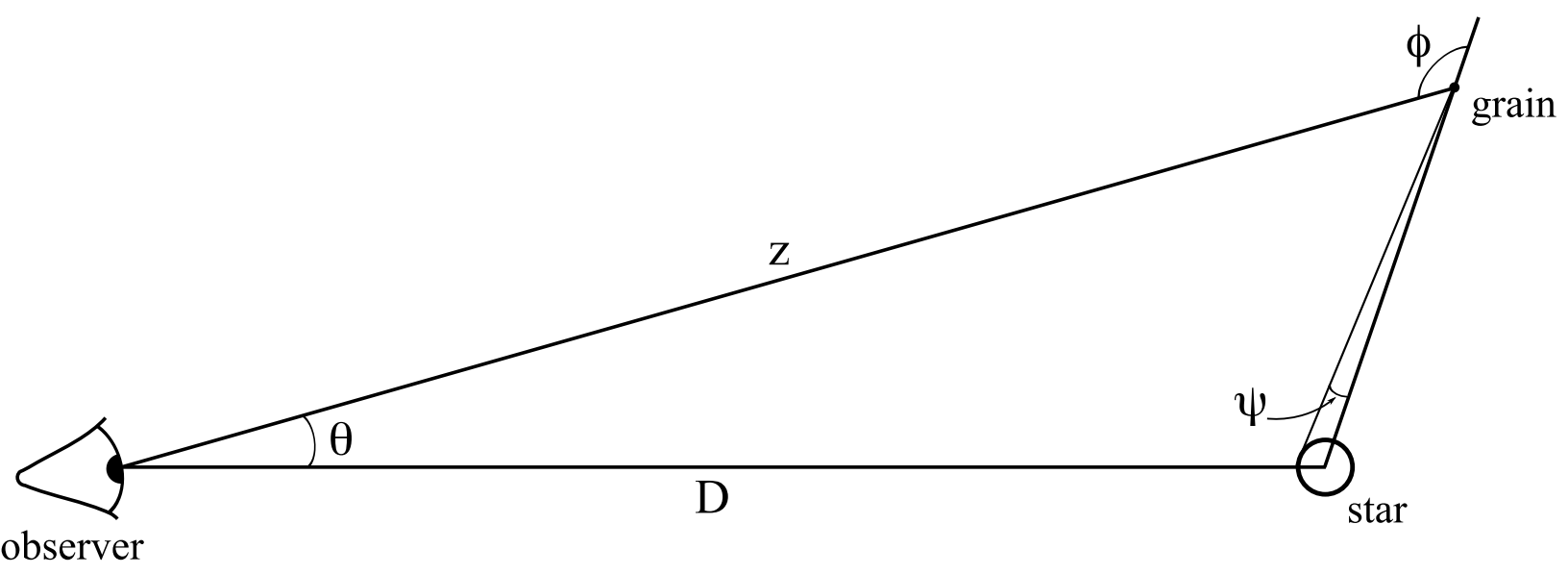

Fig. 1. - Sketch of the scattering geometry for a star at distance $D$. A representative interstellar grain lies along a line of sight offset from the star by an angle $\theta$ and is at a distance $z$. The star subtends an angle $2 \psi$ as seen from the grain. Stellar photons reach our line of sight on scattering through an angle $\phi$.

The integral is very nearly $\pi$ because $1 / \theta$ is almost $+\infty$ and the integrand falls off rapidly with $y$.

As a specific case we take the Sun observed from a distance of $100 \mathrm{pc}$ at wavelength $547 \mathrm{~nm}$ through a uniform interstellar medium with $0.1 \mathrm{H}_{\text {atoms }} \mathrm{cm}^{-3}$, similar to the densities found in the solar neighborhood (Bruhweiler \& Kondo 1982; Slavin \& Frisch 2002). For Milky Way grains, the total opacity $\chi_{\lambda} \rho=4.9 \times 10^{-22} \mathrm{~cm}^{2} \mathrm{H}^{-1} \times 0.1 \mathrm{H} \mathrm{cm}^{-3}=4.9 \times 10^{-23}$ $\mathrm{cm}^{-1}$ and albedo $\omega_{\lambda}=0.66$ (Draine 2003). The optical depth to the star $\chi_{\lambda} \rho D \approx 10^{-2}$. The predicted surface brightness ratio at an angular separation $\theta$ is

$$
\frac{I_{\lambda}(\theta)}{I_{\lambda}^{*}}=\frac{\pi \chi_{\lambda} \rho \omega_{\lambda} r_{*}^{2}}{4 D \theta}=8 \times 10^{-16}\left(\frac{n_{H}}{0.1 \mathrm{~cm}^{-3}}\right)\left(\frac{100 \mathrm{pc}}{D}\right)\left(\frac{0.1^{\prime \prime}}{\theta}\right)
$$

and falls off inversely with the separation. The integrated flux within an angle $\theta_{\max }$ is proportional to the optical depth,

$$
\frac{F_{\lambda}\left(\theta_{\max }\right)}{F_{\lambda}^{*}}=8 \times 10^{-8}\left(\frac{n_{H}}{0.1 \mathrm{~cm}^{-3}}\right)\left(\frac{D}{100 \mathrm{pc}}\right)\left(\frac{\theta_{\max }}{1^{\prime \prime}}\right)
$$

and the flux within one arcsecond is eighty times greater than the nominal threshold $10^{-9} F_{\lambda}^{*}$ for detection by the proposed Terrestrial Planet Finder Coronagraph. 


\section{ANISOTROPIC SCATTERING}

Interstellar grains scatter visible light anisotropically. We treat this effect using a standard model for grains in the diffuse interstellar medium of the Milky Way, with the phase function $\Phi_{\lambda}(\phi)$ computed from Mie theory by Draine (2003). Forward scattering is moderately preferred and at the chosen wavelength of $547 \mathrm{~nm}$, each event has a $50 \%$ chance of deflecting the photon through an angle less than $43^{\circ}$. The interstellar medium is uniform with the same density as in section 3. The transfer equation 2 is numerically integrated along the line of sight to the observer using a fourth-order Runge-Kutta method. We have checked that the method reproduces the analytic solution given by equation 6 in the case of isotropic scattering. Including the preferential forward scattering yields a halo $48 \%$ brighter. The results for a solar analog star at a distance of $100 \mathrm{pc}$ are shown in figure 2. Also shown for comparison is a solar analog exozodiacal cloud from appendix 1.B of Levine et al. (2006). The light scattered from the interstellar grains is brighter than that from the exozodiacal cloud outside 50 milliarcsec. The exozodiacal cloud is inclined $60^{\circ}$ from face-on and its brightness was calculated including anisotropic scattering.

Much of the interstellar scattered light comes from grains passing close by the star, due to the inverse square falloff of the stellar illumination. Most comes from grains whose distance from the star is less than ten times the projected separation in the plane of the sky. The preferential forward scattering has a smaller effect, making the dust in front of the star contribute more than the dust at the same distance behind. The contributions from dust at different positions along a line of sight passing 0.1 arcsecond from a star at $100 \mathrm{pc}$ are shown in figure 3. Overall, the scattered light halo is most sensitive to the distribution of interstellar dust in the vicinity of the illuminating star.

Measurements and models of the optical properties of the dust in the diffuse interstellar medium allow a range of values for the albedo and the degree of forward scattering (Gordon 2004). The Draine (2003) model used above lies near the middle of the range in both the albedo and the scattering asymmetry as measured by the mean cosine scattering angle $g=\langle\cos \phi\rangle$ at $547 \mathrm{~nm}$. The appearance of the halo is qualitatively independent of the dust properties over the allowed range. The halo brightness is simply proportional to the albedo (equation (6)). The scattering asymmetry affects both the halo brightness and the location of the grains giving most of the light. We examine the dependence by re-computing the halo using a standard Henyey-Greenstein phase function with asymmetry parameters up to the maximum $g=0.8$ consistent with the range of the measurements. Half the photons are deflected through angles less than $19^{\circ}$. The resulting halo is 2.19 times brighter than with

the Draine (2003) phase function. The fraction of the light 0.1 arcsecond from the star that is scattered by grains lying more than $200 \mathrm{AU}$ from the star rises from $7 \%$ to $21 \%$. A star 


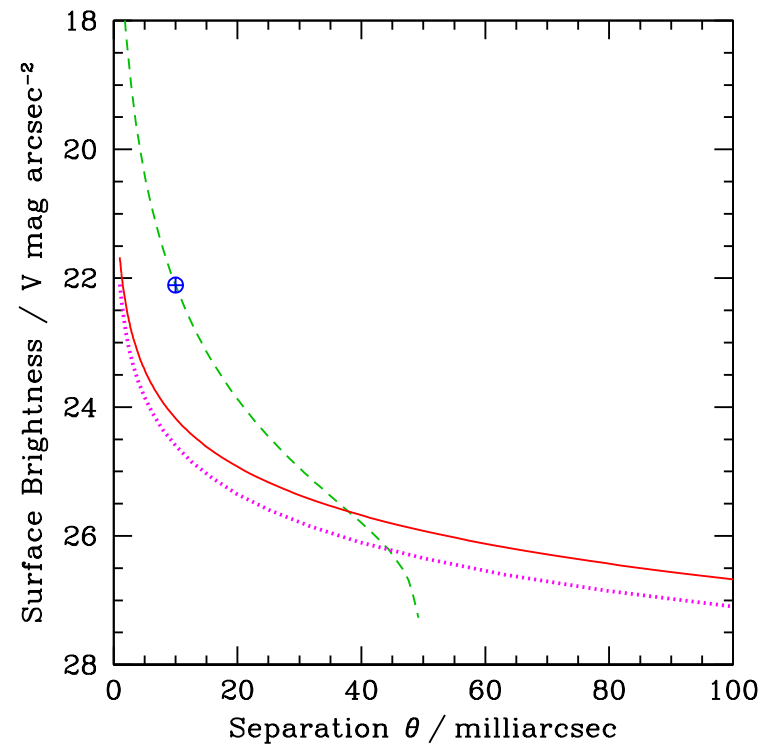

Fig. 2.- Surface brightness versus separation from the star for a solar analog exozodiacal cloud (dashed curve) and uniformly-distributed interstellar grains, scattering either isotropically (dotted line) or anisotropically according to the Draine (2003) phase function (solid line). A crossed circle marks the approximate angular separation and surface brightness of an exo-Earth at the nominal angular resolution of the Terrestrial Planet Finder Coronagraph (Levine et al. 2006). The star lies at a distance of 100 pc.

surrounded by the more forward-throwing grains has a brighter halo with a slightly lower contrast for any features arising from dust passing close by the star.

\section{PROBING ASTROSPHERES}

Sub-micron interstellar grains entering the solar system are deflected by the solar gravity, radiation pressure and solar wind electromagnetic forces (Gustafson \& Misconi 1979). The highest densities of grains 0.1 micron in radius occur where the particles pile up against the solar wind and where the magnetic forces concentrate particles over the solar magnetic poles (Grogan et al. 1996; Landgraf 2000). In this section we explore whether similar effects can be detected in the scattered light halos around other stars. 


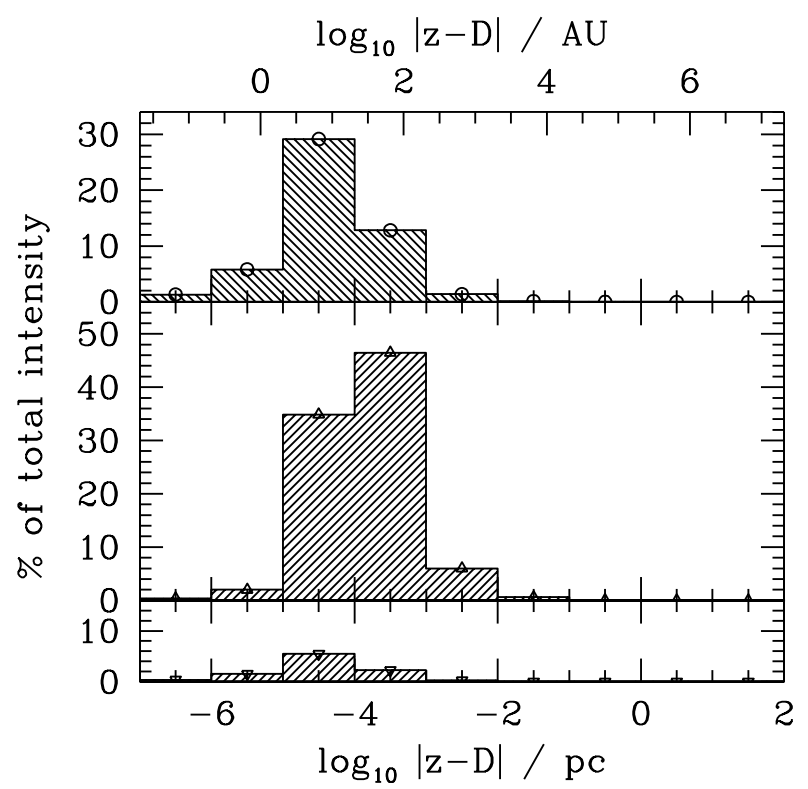

Fig. 3.- Contribution to the surface brightness from grains at different locations along the line of sight passing 100 mas or $10 \mathrm{AU}$ from the star in figure 2. The three panels show isotropic scattering (top) and anisotropic scattering by grains in front of (center) and behind the plane containing the star (bottom). The horizontal axes give the distance from the star plane in AU (above the chart) and parsecs (below). Most of the light is scattered into our line of sight by grains within $100 \mathrm{AU}$ of the star.

\subsection{Stellar Wind Model}

The star is taken to be a solar analog and its wind is modeled following Gustafson \& Misconi (1979), Grogan et al. (1996) and Landgraf (2000) by applying the expanding corona model of the solar wind magnetic field (Parker 1958). The gas streaming outward from the star draws out the stellar magnetic field lines so that near the star the field is approximately radial. The more distant field lines are dragged into the shape of an Archimedean spiral as the star rotates. The radial and azimuthal components of the field are

$$
B_{r}=B_{0} / r^{2}
$$

and

$$
B_{\phi}=-B_{0} \omega b^{2}(r-b) \sin a / V_{s w} r^{2},
$$

where $\omega$ is the stellar angular velocity, $a$ is the astrocentric colatitude angle, $V_{s w}$ is the stellar wind speed (chosen as $400 \mathrm{~km} \mathrm{~s}^{-1}$ ) and $b$ is the distance at which the reference field strength 
$B_{0}$ is taken. The Lorentz force is then

$$
\vec{F}_{L}=\frac{q}{c}\left[\left(\vec{V}_{g} \times \vec{B}\right)-\left(\vec{V}_{s w} \times \vec{B}\right)\right]
$$

where $q$ is the grain charge and $V_{g}$ the grain speed. The grain charge $q$ is given by

$$
q=4 \pi \varepsilon_{0} U s
$$

where $s$ is the grain radius, $\varepsilon_{0}=8.86 \times 10^{-12} \mathrm{CV}^{-1} \mathrm{~m}^{-1}$ is the permittivity of free space and $U$ is the grain surface potential. Dust in the stellar wind cavity is expected to be positively charged due to the dominance of stellar photoionization over the competing mechanism of the 'sticking' of stellar wind electrons (Mukai 1981). Since the stellar wind density roughly follows an inverse square law decrease with astrocentric distance, particles are charged to $+5-10$ volts with little distance variation. We adopt a surface potential $U$ of +5 volts. Notice that the dominant electric part in equation 10 is proportional to $B_{\phi}$ and therefore to $r^{-1}$. This can make the instantaneous Lorentz force dominate on small grains at large astrocentric distances. The ballerina model proposed by Alfvén (1977) gives an explanation for solar system spacecraft measurements which indicate that near the ecliptic plane the interplanetary magnetic field is directed inward in certain regions and outward in others. In this picture a current sheet separates plasma from either hemisphere carrying fields of opposite polarity. As the sheet rotates with the Sun the small up and down displacements in the sheet, similar to the wave motion of the skirt of a spinning ballerina, explain the observed effect. We use a simplified model of this phenomenon to describe the magnetic field strength averaged over a stellar rotation by the inclusion of a current sheet which changes its tilt angle $\varepsilon$ to the stellar equatorial plane at a constant angular velocity of $(1 / 11) \pi$ radians per year over the stellar magnetic activity cycle (Gustafson 1994), so that the sheet completes one $360^{\circ}$ rotation in 22 years. The averaged field at latitude $A$ is then a fraction $2 \arcsin (\tan A / \tan \varepsilon) / \pi$ of the unipolar field. The line of nodes defined by the intersection of the current sheet and stellar equatorial plane will rotate through $360^{\circ}$ in one 27 day stellar rotation. The effect of radiation pressure is to reduce the stellar gravitational force, the magnitude of the radiation pressure being given by

$$
P_{p r}=\frac{S}{c} Q_{p r}
$$

where $S$ is the flux, $c$ is the speed of light and $Q_{p r}$ is the radiation pressure efficiency of the particle.

\subsection{Particle Properties}

Grains larger than one micron are rare in the diffuse interstellar medium according to extinction curve fitting results (Mathis, Rumpl \& Nordsieck 1977; Draine \& Lee 1984; 
Weingartner \& Draine 2001). Grains smaller than 0.01 micron have little optical crosssection and furthermore are largely excluded from the heliosphere by magnetic forces due to their high charge-to-mass ratios. Grains in the 0.1 micron size range experience a radiation pressure force larger than the gravitational force but nevertheless are able to penetrate deep into the solar system (Grogan et al. 1996). Most of the scattered light from interstellar grains passing through the astrosphere of a solar analog star is likely due to grains about 0.1 micron in size. We therefore concentrate on grains with radii between 0.02 and 0.3 microns.

The ratio $\beta$ of the radiation pressure force to the gravitational force may be expressed in terms of $Q_{p r}$ as

$$
\beta=\frac{5.7 \times 10^{-5} Q_{p r}}{s \rho},
$$

where $\rho$ is the density of the particle (Burns et al. 1979). The optical efficiencies of the grains are found from Mie theory. We assume astronomical silicate composition and optical constants (Laor \& Draine 1993). Clearly, the particle spatial distributions will vary with the particle size; for example, the gravitational force will dominate for larger particles, whereas the interaction with the magnetic field will become increasingly important at the small particle end of the size range. The distribution of particles will also depend on the stellar magnetic cycle, so that the particle positions will represent a 'snapshot' in time rather than an equilibrium configuration.

\subsection{Dynamical Calculations}

Taking into account gravity, radiation pressure and the stellar magnetic field model outlined above, we follow the trajectories of a uniform distribution of dust particles at infinity as they approach the star from the upstream direction at a speed of $26 \mathrm{~km} \mathrm{~s}^{-1}$. The equations of motion are integrated at small intervals of time and the position, speed and acceleration of the particles are calculated. The local properties of the stellar wind and magnetic field are updated as the integration progresses. We continue the integration until the heliosphere analog is filled with dust particles and stop the run at a given phase of the stellar magnetic field cycle with the magnetic field orientation matching that of the Sun in the year 1990 .

The dust density is then calculated by binning the particles in cubes $2 \mathrm{AU}$ on a side and normalizing to the upstream interstellar density. The resulting distributions are broadly similar for particles 0.02 to $0.3 \mu \mathrm{m}$ in radius. The distribution of $0.1 \mu \mathrm{m}$ grains is shown in figure 4. The particles are pushed by the magnetic forces into the equatorial current sheet, and pile up 10 AU upstream from the star (left panel), where the density reaches six times 


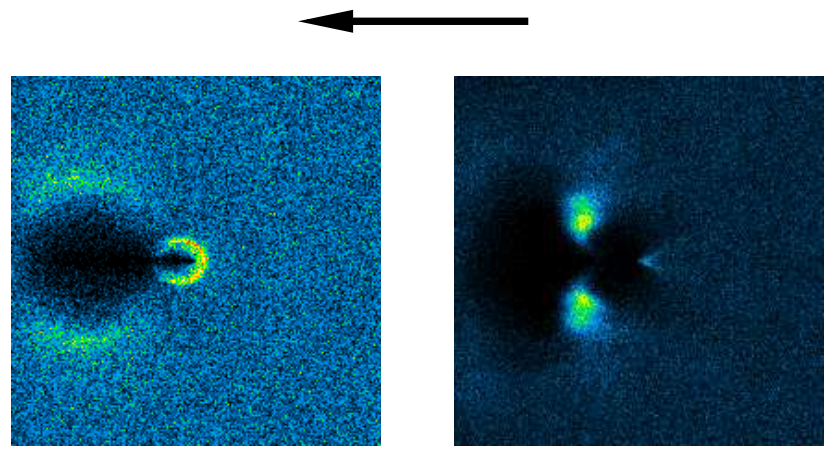

Fig. 4.- Interstellar dust density near a Sun-like star. The star is centered in each image and the dust enters from the right, as shown by the arrow. At left is a slice through the equatorial plane and at right is the polar plane containing the velocity vector. The images are $400 \mathrm{AU}$ on a side. The color scale is linear between zero (black) and the maximum density (red). Focusing by the stellar gravity, radiation and magnetic forces enhances the density of the $0.1 \mu \mathrm{m}$ particles up to 6 times at left and 15 times on the right.

that in the interstellar medium. The greatest density enhancement of all is a factor fifteen and occurs in two fins over the magnetic poles and 60 AU downstream from the star (right panel). These result from the focusing of the grains by the inclined magnetic fields off the equatorial plane.

\subsection{Synthetic Images}

Synthetic images of the scattered starlight are constructed by solving the transfer equation 2 to find the intensity on a grid of sky points, treating the anisotropic scattering as described in section 4 with the Draine (2003) Mie theory phase function. No exozodiacal cloud is included. Selected results are shown in figures [5] and 6. In addition to an overall deficit of scattered light relative to the case of uniformly-distributed dust, several features produced by the magnetic field are clearly visible, including the density enhancements in the equatorial disk and the polar fins.

\section{CONCLUSIONS}

We have shown that stars appear surrounded by halos of light scattered by interstellar dust. The halos are bright enough for detection with the proposed Terrestrial Planet Finder 

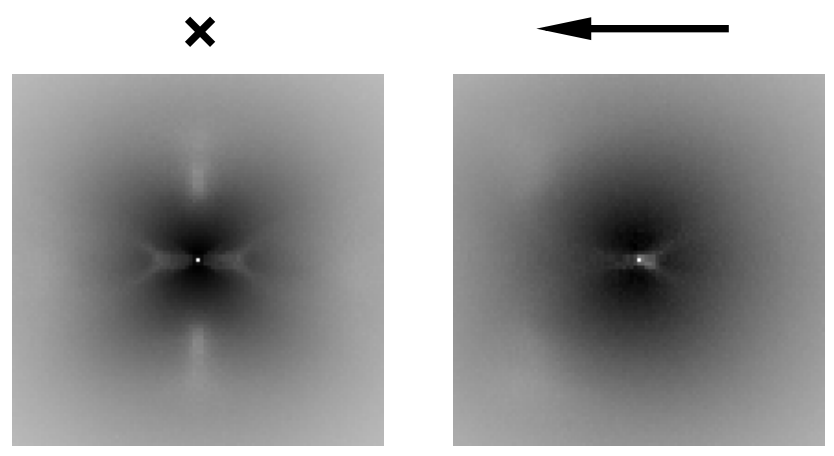

Fig. 5.- Model images of the starlight scattered by $0.1 \mu \mathrm{m}$ interstellar grains around a Sun-like star at a distance of 100 parsec. The star is viewed looking upstream (left panel) and from the side (right panel). The cross and arrow indicate the flow of the approaching grains. The images are 2 arcsec or 200 AU across, and were divided by the profile for uniformly-distributed dust. Dark regions show a deficit of scattered light where magnetic and radiation forces exclude particles near the star. The gray scale is linear between the minimum brightness ratio of about 0.1 (black) and the maximum of unity (white).

Coronagraph around stars at distances of $100 \mathrm{pc}$ and greater. The halo reveals the distribution of dust in the vicinity of the star, providing opportunities to probe the interstellar medium and measure the properties of the stellar wind. Particles of different sizes have different distributions, with larger particles approaching the star most closely (Grogan et al. 1996). This size-sorting can potentially be used to measure the distribution of grain sizes at selected locations in the interstellar medium and to resolve whether particles larger than one micron are present in significant numbers, as suggested by dust impact detector data from the Ulysses and Galileo spacecraft (Frisch et al. 1999; Weingartner \& Draine 2001). Since the grains are deflected by stellar wind magnetic forces, the features in the scattered light halo can be used to estimate the wind speed and the relative motion between the star and the surrounding interstellar medium. The results will complement the wind mass loss rates estimated using the Lyman- $\alpha$ absorption by the gas collected near stellar wind bow shocks (Wood et al. 2005). The scattering halos will make it possible in addition to determine the wind magnetic field strength and orientation (figure 5) and, by observations spanning a decade or so, the variation over the stellar activity cycle (Landgraf 2000). Imaging the halos will yield fresh information about the winds of stars with a range of ages and could lead to a better understanding of stellar spin histories (Bouvier et al. 1997) and the long-term evolution of stellar activity.

This work was carried out at the Jet Propulsion Laboratory, California Institute of 


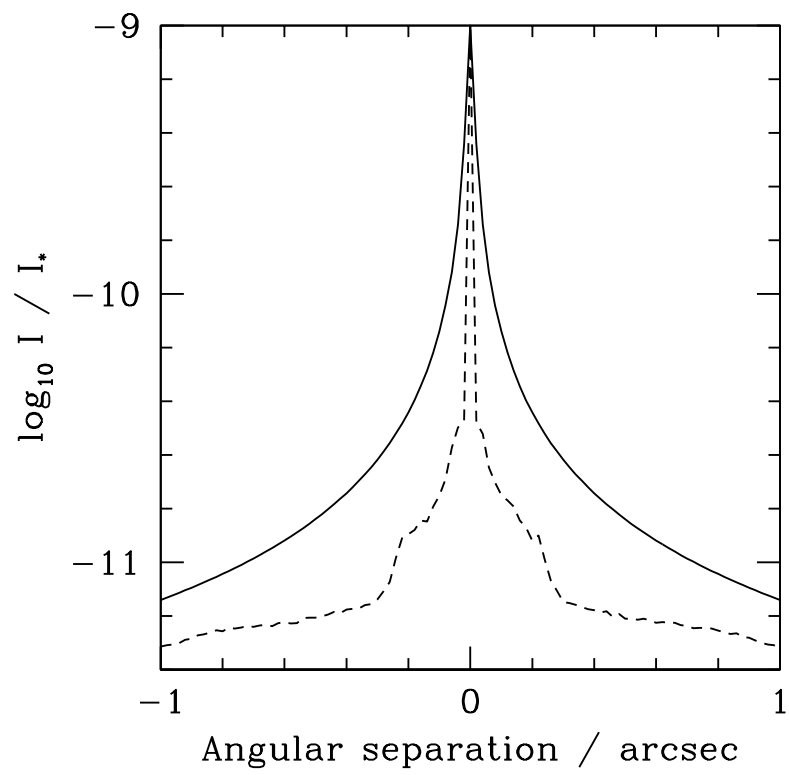

Fig. 6. - Model profiles of the starlight scattered by $0.1 \mu \mathrm{m}$ interstellar grains around a Sun-like star at a distance of 100 parsec. The solid line is for uniformly-distributed dust. The dashed line shows the effects of stellar gravity, radiation and magnetic forces pushing aside dust near the star, and is a horizontal cut through the center of figure 5, left panel. The vertical axis is the count rate per pixel on a logarithmic scale, in units of the stellar count rate. The pixels are 20 milliarcsec on a side.

Technology with support from the internal Research and Technology Development Fund Innovative Spontaneous Concepts Program.

\section{REFERENCES}

Alfvén, H. 1977, Rev. Geophys. Space Phys. 15, 271

Bouvier, J., Forestini, M., \& Allain, S. 1997, A\&A, 326, 1023

Bruhweiler, F. C., \& Kondo, Y. 1982, ApJ, 259, 232

Burgh, E. B., McCandliss, S. R., \& Feldman, P. D. 2002, ApJ, 575, 240

Burns, J. A., Lamy, P. L., \& Soter, S. 1979, Icarus, 40, 1

Calzetti, D., Bohlin, R. C., Gordon, K. D., Witt, A. N., \& Bianchi, L. 1995, ApJ, 446, 97 
Draine, B. T. 2003, ApJ, 598, 1017

Draine, B. T., \& Lee, H. M. 1984, ApJ, 285, 89

Draine, B. T., \& Tan, J. C. 2003, ApJ, 594, 347

Fitzgerald, M. P., Stephens, T. C., \& Witt, A. N. 1976, ApJ, 208, 709

Frisch, P. C., et al. 1999, ApJ, 525, 492

Gordon, K. D. 2004, in ASP Conf. Ser. 309, Astrophysics of Dust, ed. A. N. Witt, G. C. Clayton, \& B. T. Draine (San Francisco: ASP), 77

Grogan, K., Dermott, S. F., \& Gustafson, B. A. S. 1996, ApJ, 472, 812

Grogan, K., Dermott, S. F., \& Durda, D. D. 2001, Icarus, 152, 251

Grün, E., et al. 1994, A\&A, 285, 915

Gustafson, B. A. S., \& Misconi, N. Y. 1979, Nature 282, 276

Gustafson, B. A. S. 1994, Annu. Rev. Earth Planet. Sci. 22, 553

Hurwitz, M., Bowyer, S., \& Martin, C. 1991, ApJ, 372, 167

Landgraf, M. 2000, J. Geophys. Res., 105, 10303

Laor, A., \& Draine, B. T. 1993, ApJ, 402, 441

Levine, M., Shaklan, S., \& Kasting, J. 2006, Terrestrial Planet Finder Coronagraph - Science and Technology Definition Team Report, publication D-34923 of the Jet Propulsion Laboratory, California Institute of Technology

Mathis, J. S., Rumpl, W., \& Nordsieck, K. H. 1977, ApJ, 217, 425

Mathis, J. S., Cohen, D., Finley, J. P., \& Krautter, J. 1995, ApJ, 449, 320

Mukai, T. 1981, A\&A, 99, 1

Murthy, J., \& Henry, R. C. 1995, ApJ, 448, 848

Parker, E. N. 1958, ApJ, 128, 664

Slavin, J. D., \& Frisch, P. C. 2002, ApJ, 565, 364

Smith, R. K., \& Dwek, E. 1998, ApJ, 503, 831 
Weingartner, J. C., \& Draine, B. T. 2001, ApJ, 548, 296

Witt, A. N., Oliveri, M. V., \& Schild, R. E. 1990, AJ, 99, 888

Witt, A. N., Petersohn, J. K., Bohlin, R. C., O'Connell, R. W., Robert, M. S., Smith, A. M., \& Stecher, T. P. 1992, ApJ, 395, L5

Witt, A. N., Friedmann, B. C., \& Sasseen, T. P. 1997, ApJ, 481, 809

Witt, A. N., Smith, R. K., \& Dwek, E. 2001, ApJ, 550, 201

Wood, B. E., Müller, H.-R., Zank, G. P., Linsky, J. L., \& Redfield, S. 2005, ApJ, 628, L143 